\title{
Modeling the Traffic Flow of the Urban Signalized Intersection with a Straddling Work Zone
}

\author{
Liling Zhu, ${ }^{1,2}$ Bingmei Jia $\mathbb{D}^{2},{ }^{2}$ Da Yang $\mathbb{D}^{2,3}$ Yuezhu Wu, ${ }^{2}$ Guo Yang, ${ }^{2}$ Jingang Gu, ${ }^{3}$ \\ Hongtong Qiu, ${ }^{3}$ and Qian Guo $\mathbb{i D}^{2}$ \\ ${ }^{1}$ School of Business, Sichuan Normal University, No. 1819, Section 2, Chenglong Avenue, Chengdu 610101, China \\ ${ }^{2}$ School of Transportation and Logistics, \\ National Engineering Laboratory of Integrated Transportation Big Data Application Technology, \\ National United Engineering Laboratory of Integrated and Intelligent Transportation, Southwest Jiaotong University, \\ No. 999 Piduquxianlu, Chengdu 611756, China \\ ${ }^{3}$ Traffic Management Research Institute, Ministry of Public Security, Wuxi 214151, China
}

Correspondence should be addressed to Bingmei Jia; jiabingmei@my.swjtu.edu.cn and Da Yang; yangd8@gmail.com

Received 14 January 2020; Revised 26 July 2020; Accepted 20 August 2020; Published 9 September 2020

Academic Editor: Jaeyoung Lee

Copyright (c) 2020 Liling Zhu et al. This is an open access article distributed under the Creative Commons Attribution License, which permits unrestricted use, distribution, and reproduction in any medium, provided the original work is properly cited.

Work zones widely exist on urban roads in many countries and have a significant negative impact on traffic. Few studies have focused on modeling the traffic flow of the work zone on the urban arterials, especially on the work zone at the intersections. In this paper, a microscopic model based on the social force theory for the traffic flow of the intersection with a specific work zone, called straddling work zone, is proposed. The model can capture the no lane division and irregular boundary characteristics of the traffic of the intersection with a straddling work zone and also can reflect the interaction of the intersection traffic flows from the two opposite directions. The proposed model is calibrated and validated using the real work zone data, and the results display that the MARE values are all less than $10 \%$. The factors affecting the traffic flow in the straddling work zone are analyzed through simulation. Our study reveals that the distance from the lower edge of the work zone to the median divider of the road and the proportion of large vehicles in the work zone have the greatest impact on the signalized intersection, which provides a reference for the future traffic control at the intersection with the straddling work zone.

\section{Introduction}

Work zones exist widely on both freeways and city arterials in many countries and have a significant negative impact on the road traffic. According to the existing studies, work zones will cause heavy traffic congestion and more traffic accidents [1-7]. It is necessary to look for the ways of reducing the influence of work zones on traffic.

Although numerous studies have been conducted on the traffic problems of work zones, they mainly focused on the traffic problems of freeway work zones, such as traffic safety [8], road capacity [9], and traffic delays [10]. The studies on the urban arterials work zones are very limited [11-15]. There are many work zones located on both road segments and intersections of the cities in many countries, and this paper focuses on the intersection work zones on city arterials. The intersection work zones can be classified into two types: the island work zone and the straddling work zone (see Figure 1). The island work zone refers to the work zone that is located entirely within the area of the intersection (see Figure 1(a)). The straddling work zone refers to the work zone that straddles both an intersection and a road segment (see Figure 1(b)). Yang et al. [7] had proposed a traffic flow model for the island work zone and analyzed the traffic flow characteristics. However, the traffic flow model for the straddling work zone has not been proposed yet.

Therefore, this paper attempts to propose a microscopic traffic flow model for the straddling work zone. As shown in Figure 1(b), in the straddling work zone, the part of the original space of the intersection is occupied by the 


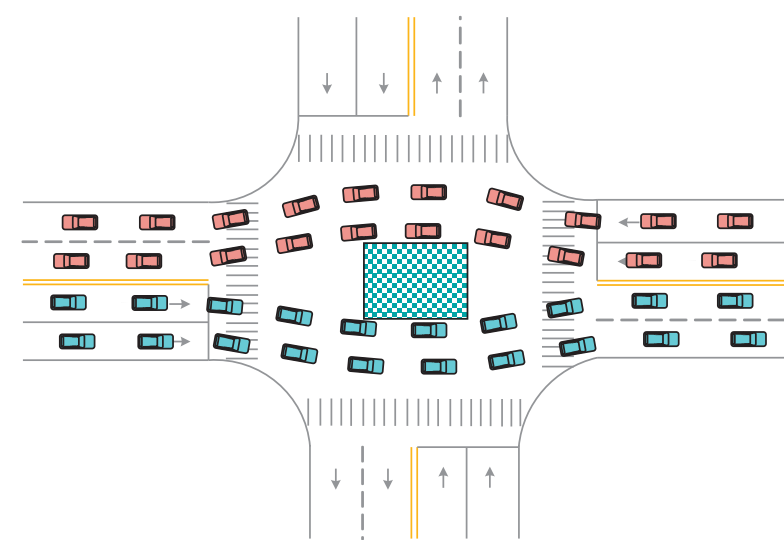

(a)

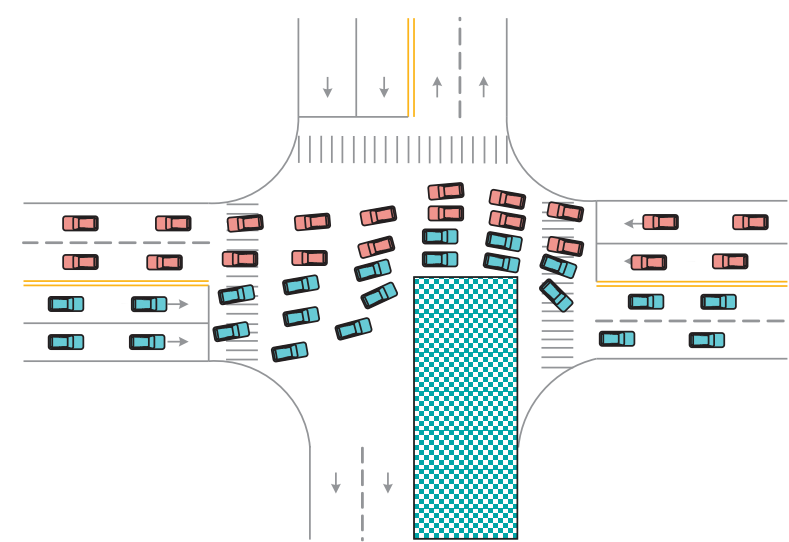

(b)

FIgURE 1: Two types of the intersection work zone. (a) Island work zone. (b) Straddling work zone.

straddling work zone and the passable space is seriously reduced, which makes the traffic flow of the straddling work zone exhibit the different characteristics with the normal traffic. First, the area of the straddling work zone has no lane divisions, so the vehicles cannot move straightly in such an area. Second, the boundary of the passable area of the intersection becomes more irregular, which causes more conflicts among vehicles. The existing microscopic traffic flow models, such as the car-following models and lanechanging models, cannot capture the above characteristics, for the reason that they were designed for the lane-based traffic flow in a regular passable area $[16,17]$. In addition, the traffic flow of the straddling work is also different from that of the island work zone. In the island work zone, the two traffic flows from the opposite directions are separated by the work zone (as shown in Figure 1(a)). Meanwhile, in the straddling work zone, the traffic flow has strong interaction with the oncoming traffic flow. Thus, the proposed traffic flow model for the island work zone by the authors in [7] cannot be applied to the traffic flow of the straddling work zone, and the new model is necessary. In our research, the social force theory is applied to model the traffic flow of the straddling work zone, and we construct a non-lane-based model to overcome the shortcomings of the conventional microscopic traffic flow models, which describes the traffic flow of the straddling work zone.

The rest of the paper is organized as follows. Section 2 reviews the existing studies on work zones and social force theory. Section 3 presents the proposed model for the traffic flow of the straddling work zone. Section 4 calibrates and validates the proposed model using the collected data. Section 5 discusses the simulation and analysis based on the proposed model. Section 6 concludes the paper and discusses the future work.

\section{Literature Review}

The study on work zones located on city arterials is receiving more and more attention. Some works focused on the work zones on arterial segments between the two intersections. Sadegh et al. [18] applied car-following and lane-changing models to describe the traffic flow of the work zone on the arterial segment and further developed a simulation tool named ARTWORK to evaluate the traffic control systems for the condition that lanes are closed in the vicinity of signalized intersections. Joseph et al. [19] developed a tool to analyze and evaluate the traffic in the case that a lane is closed between the two signalized intersections, which was named WZATA. Tsyganov et al. [20, 21] collected traffic crash data in work zones on urban arterials in Austin, San Antonio, and Houston metropolitan areas to give suggestions to improve the traffic control in an urban arterial work zone. Heaslip et al. [22] used simulation method to estimate the capacity of work zones on arterial segments with consideration of various factors including the percent of leftturning vehicles, the distance between the work zone and the downstream intersection, and the g/C (effective green to cycle length) of each lane group. They drew the conclusions that work zones on arterial segments affected the capacity of the entire arterial, and the results were highly impacted by the distance between the work zone and the downstream intersection. Hou et al. [15] proposed four machine-learning-based models: random forest, regression tree, multilayer feed-forward neural network, and nonparametric regression. Two sets of data for both expressway and urban road intersection were collected to predict the traffic flow of the urban work zone.

Some studies explored the work zones located on intersections and investigated the signal design improvement [13], safety issue [11], and work zone layout strategy [12]. An overview [23] qualitatively suggested several methods of improving the signal design of an intersection with work zone, such as adjustment of signal phase and time, shortening of cycle lengths, and accommodation of pedestrians. However, the suggested methods lacked real data support and scientific analysis. Li and Bai [11] studied the crashes in intersection work zones and found that the number of crashes happening at intersections was much less than the number of crashes happening at nonintersections, which may be attributed to vehicles' relative lower speed at intersections. Shaaban and Elnashar [12] discussed the issues of the intersection work zones and presented a work zone 
layout strategy at an intersection. Yang et al. [14] collected the intersection work zone data and proposed a linear regression model to estimate the capacity of the urban signal intersection with work zone for the first time. However, their model was a statistical model, and the mechanism of how work zones impact the intersection traffic flow was still unknown. Recently, Yang et al. [7] focused on a specific type of work zone which is located within the area of the intersection, namely, the island work zone, and proposed a novel model for the traffic flow of the island work zone based on the social force theory. They evaluated the proposed model by field work zone data, while the results indicated that the model obtained high performance.

In summary, the majority of the existing studies on arterial work zones focused on the work zones on road segments. Few studies on work zones at intersections model the traffic flow of the straddling work zone. Therefore, a new model is needed to better investigate the traffic flow characteristics of the straddling work zone.

\section{Model Development}

3.1. Driver Behavior Analysis of the Straddling Work Zone. When a straddling work zone exists at an intersection, the driver behaviors are obviously changed. We extracted the five types of driver behavior: the free-driving behavior, following behavior, merging behavior, diffusing behavior, and shuttling behavior. The free-driving behavior means the vehicles can pass the intersection freely without any obstructions by work zones or other vehicles. This type of behavior belongs to the leading vehicles of the queue before the stop line. The following behavior is adopted by the vehicles that have to follow the preceding vehicles. The merging behavior refers to the situation where a vehicle bypasses the work zone to merge into the main stream. The diffusing behavior happens after the vehicles pass through the work zone area. It describes the behavior that vehicles diffuse to a wider space behind the work zone. The shuttling behavior belongs to the aggressive vehicles that prefer to obtain a faster speed or a more forward position. The merging behavior, diffusing behavior, and shuttling behavior are the three types of driver behaviors that are unique for the traffic of the intersection with a work zone.

In addition, when a straddling work zone exists, the trajectories of vehicles passing the intersection will be changed significantly. When the traffic light turns to green, the queuing vehicles behind the stop line are released. However, the vehicles cannot move forward straightly. They are forced to move following a curve to bypass the straddling work zone. Meanwhile, the vehicles from the opposite direction are simultaneously released when the green light is on. The trajectories of the vehicles are squeezed toward the space originally designed for the oncoming vehicles due to the existence of the work zone. To the end, at the intersection with a straddling work zone, the vehicles from the two opposite directions have strong interactions. In summary, the existence of a straddling work zone has seriously affected the passing behavior of vehicles at the intersection. Therefore, a new model that can capture the traffic flow characteristics of the straddling work zone is needed.

\subsection{Improved Social Force Model for the Straddling Work} Zone. The proposed social force system for the vehicles in a straddling work zone is shown in Figure 2. In the paper, the six types of social force are proposed to capture the driver behaviors of vehicles at an intersection with a straddling work zone: the self-driven force, following force, passablegap force, repulsive force, boundary force, and friction force.

3.2.1. Self-Driven Force. When the leading vehicles in the queue start from the stop line, they attempt to accelerate to a desired speed and maintain the desired speed to pass the intersection. The leading vehicles adopt the free-driving behavior. A self-driven force is acted on the leading vehicle to capture its driver characteristic. The self-driven force pointing to the exit of the intersection is proportional to the speed difference of the actual speed and the desired speed and cannot exceed the maximum acceleration. The force can make the vehicle adjust its speed in each adaption time to reach the allowed maximum speed of the intersection. The equation of the self-driven force is as follows:

$$
\vec{F}_{i}^{s}=\min \left(a_{\max }, \frac{\left[v_{i}^{0}(t) \vec{e}_{i}(t)-\vec{v}_{i}(t)\right]}{\tau_{i}}\right),
$$

where $\vec{F}_{i}^{s}$ denotes the self-driven force, $a_{\max }$ denotes the maximum acceleration of the vehicle $i, v_{i}^{0}(t)$ and $\vec{v}_{i}(t)$, respectively, denote the desired and actual velocities of the vehicle $i, \tau_{i}$ denotes the reaction time of the vehicle $i$, and $\vec{e}_{i}(t)$ denotes the desired direction of the vehicle $i$ at the time $t$, which can be derived from the current position $x_{0}(t)$ and the desired position $x_{d}(t)$ of the vehicle $i$, and has the following expression:

$$
\vec{e}_{i}(t)=\frac{x_{d}(t)-x_{0}(t)}{\left\|x_{d}(t)-x_{0}(t)\right\|}
$$

3.2.2. Following Force. When a vehicle is forced to follow its preceding vehicle, it exhibits the following behavior and travels forward adopting a safe speed. To reflect the characteristic of the following behavior, the following force is acted on the following vehicle, which points to its preceding vehicle. In the following force, when the speed of the following vehicle is less than a safe speed, it makes the vehicle accelerate; otherwise, the vehicle will decelerate. The equation of the following force is as follows:

$$
\vec{F}_{i}^{f}=\min \left\{a_{\max }, \frac{v_{s}(t)-v_{i}(t)}{\tau_{i}}\right\} \cdot \vec{n}_{i}^{f},
$$

where $\vec{F}_{i}^{f}$ denotes the following force, $\vec{n}_{i}^{f}$ denotes the standard vector pointing from the vehicle $i$ to its preceding vehicle, and $v_{s}(t)$ denotes the safe velocity that the vehicle $i$ should keep with its preceding vehicle at the time $t$ and it can 


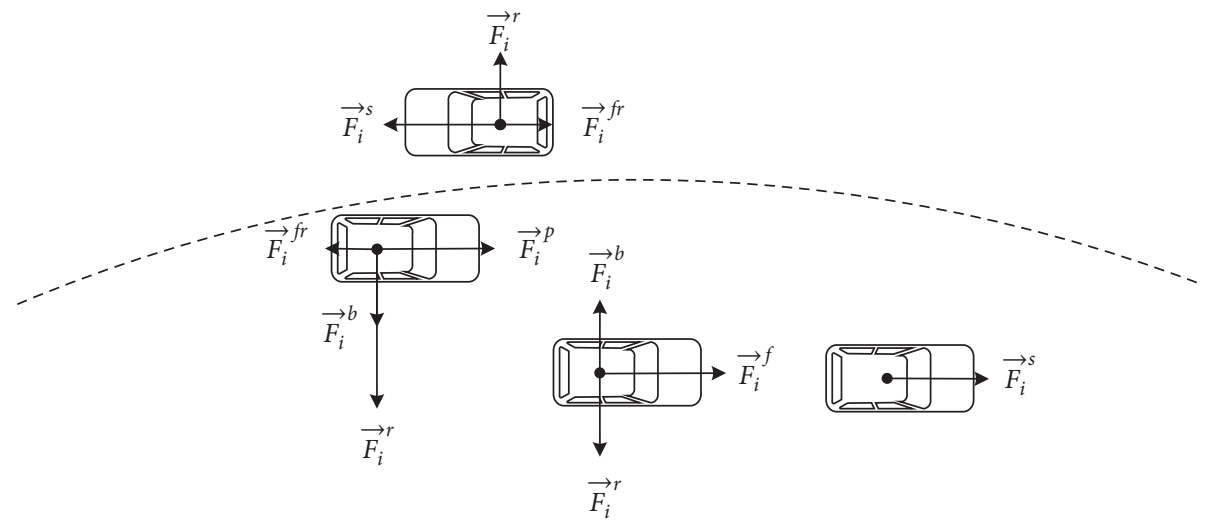

FIGURE 2: Social forces of the vehicles in the straddling work zone.

be calculated by Gipps' safety car-following model [24] as follows:

$$
v_{s}(t)=\sqrt{b_{i}^{2} \tau_{i}^{2}+b_{i}\left[\frac{2 d_{s}(t)-\tau_{i} v_{i}(t)+v_{j}^{2}(t)}{b_{j}}\right]}-b_{i} \tau_{i}
$$

where $b_{i}$ denotes the maximum deceleration of the following vehicle $i, b_{j}$ denotes the maximum deceleration of the preceding vehicle $j, d_{s}(t)$ denotes the gap between the vehicles $i$ and $j$, and $v_{j}(t)$ denotes the velocity of the preceding vehicle $j$ at the time $t$.

3.2.3. Passable-Gap Force. As shown in Figure 3, when a big gap appears ahead, the vehicle will try to enter into the gap, which is driven by a force named the passable-gap force. The passable-gap force points to the center point of the gap. In the merging, diffusing, and shuttling behaviors, a vehicle may be acted on by a passable-gap force. If the gap ahead is greater than the sum of the vehicle width and the safety side margin (e.g., $g_{c}$ in Figure 3), the passable-gap force is acted on the vehicle. The larger the gap is, the larger the value of the passable-gap force is. It should be noted that if a vehicle is acted on by a passable-gap force, it needs to compare the values of the following force and the passable-gap force. If the following force is bigger than the passable-gap force, the vehicle will follow this preceding vehicle; otherwise, it will enter into the bigger gap ahead. The passable-gap force is expressed as an exponential function of the gap $g_{c}$ as follows:

$$
\vec{F}_{i}^{p}=\min \left(a_{i}^{\max }, A^{p} \exp \left[\frac{g_{c}-\left(d_{f}+2 \Delta d\right)}{d_{f}+2 \Delta d}\right] \cdot \vec{n}_{i}^{p}\right),
$$

where $\vec{F}_{i}^{p}$ denotes the passable-gap force, $A^{p}$ denotes a parameter, $d_{f}$ denotes the width of the subject vehicle, $\Delta d$ denotes the safe side distance from the subject vehicle to the side vehicle when the subject vehicle travels through the passable gap, and $\vec{n}_{i}^{p}$ denotes the direction of passable-gap force, that is, a standard vector pointing from the front midpoint of the subject vehicle $i$ to the midpoint of the passable gap.

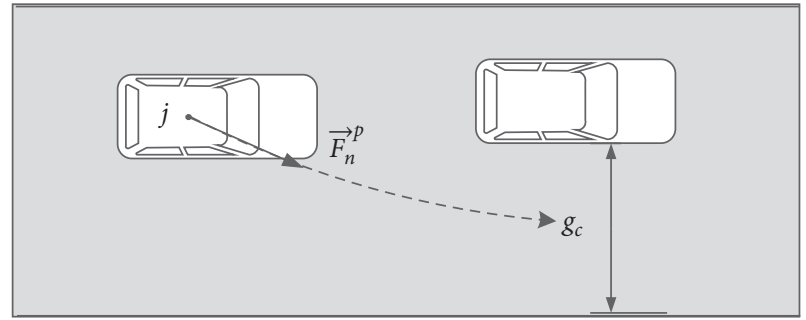

FIgURE 3: Passable-gap force of vehicles at the intersection with a straddling work zone.

3.2.4. Repulsive Force. To avoid the lateral collisions of a vehicle to the side vehicles, a force named the repulsive force is acted on the vehicle. When the distance between a vehicle and its side vehicle is more than the acting scope, the repulsive force is very small. When the distance is less than the acting scope, the repulsive force increases very fast with the decrement of the distance to ensure safety. The two vehicles are not allowed to touch each other, so the force should be of an extremely large value when the distance is very small. The repulsive force is a function of the lateral distance of two neighboring vehicles and has the following expression:

$$
\vec{F}_{i}^{r}= \begin{cases}A^{r} \cdot \exp \left[-\frac{d_{i j}-B_{r}}{B_{r}}\right] \cdot \vec{n}_{i}^{r}, & d_{i j}-\Delta d<B_{r}, \\ 0, & d_{i j}-\Delta d \geq B_{r},\end{cases}
$$

where $\vec{F}_{i}^{r}$ denotes the repulsive force, $A^{r}$ denotes the parameter, $d_{i j}$ denotes the side distance between the vehicle $i$ and the side vehicle $j, B_{r}$ denotes acting scope of the social repulsive force of the vehicle $j$, which means when the vehicle $i$ invades the acting scope of the vehicle $j$, the repulsive force will be generated, and $\vec{n}_{i}^{r}$ denotes the standard vector pointing from the vehicle $j$ to the vehicle $i$ laterally.

3.2.5. Boundary Force. Similarly, to avoid a collision to the boundary, the boundary force is also needed, and the formula is as follows: 


$$
\vec{F}_{i}^{b}= \begin{cases}A^{b} \cdot \exp \left[-\frac{d_{i b}-B_{b}}{B_{b}}\right] \cdot \vec{n}_{i}^{b}, & d_{i b}-\Delta d<B_{b}, \\ 0, & d_{i b}-\Delta d \geq B_{b},\end{cases}
$$

where $\vec{F}_{i}^{r}$ denotes the boundary force, $A^{b}$ denotes a parameter, $d_{i b}$ denotes the lateral distance from the vehicle $i$ to the boundary, and $B_{b}$ denotes the acting scope of boundary force, which means when the distance between the vehicle $i$ and the boundary is less than $B_{b}$, the boundary force will be acted on the vehicle $i . \vec{n}_{i}^{b}$ denotes the standard vector pointing from the boundary to the vehicle $i$ laterally.

3.2.6. Friction Force. To reflect the interactions between the vehicles from the two opposite directions, a friction force is added. The direction of the friction force is opposite to the driving direction of the vehicles. The friction force will produce a deceleration that is opposite to the direction of travel, causing the vehicle to slow down. It will also have a certain impact on the number of passing vehicles and the average travel time. The friction force is expressed as follows:

$$
F_{i}^{f r}= \begin{cases}A^{f r} \exp \left(\frac{l_{i j} \times d_{i j}}{2 \Delta d}\right) \Delta v_{i j} \vec{n}_{i}^{f r}, & d_{i j} \geq 2 \cdot \Delta d, \\ 0, & \text { others, }\end{cases}
$$

where $\vec{F}_{i}^{f r}$ denotes the boundary force, $A^{f r}$ denotes the parameter, $\Delta \mathrm{d}$ denotes the safe distance through which the vehicle can pass smoothly (width of the rearview mirror, etc., taking $1 / 4$ of the width of the vehicle), $l_{i j}$ denotes the longitudinal distance between the vehicle $i$ and the oncoming vehicle $j, d_{i j}$ denotes the lateral distance of the two vehicles $i$ and $j, \Delta v_{i j}$ denotes the relative speed of the vehicles $i$ and $j$, and $\vec{n}_{i}^{f r}$ denotes the standard vector pointing from the oncoming vehicle $j$ to the vehicle $i$.

In summary, the combined force acted on the leading vehicles is as follows:

$$
\vec{F}_{i}=\vec{F}_{i}^{s}+\vec{F}_{i}^{r}+\vec{F}_{i}^{b}+F_{i}^{f r} .
$$

The combined force acted on the other vehicles is as follows:

$$
\vec{F}_{i}=\max \left(\vec{F}_{i}^{f}, \vec{F}_{i}^{p}\right)+\vec{F}_{i}^{r}+\vec{F}_{i}^{b}+F_{i}^{f r} .
$$

The traffic flow model proposed in this paper is a forcebased model, and it is different from the commonly used lane-based car-following or lane-changing models, so the model has a wide range of applications. The model not only can be used to describe the regular traffic without a work zone but also can be used to reflect the characteristics of the irregular traffic, such as the unstructured road, mixed traffic, work zone traffic, and abnormal intersections.

\section{Model Calibration and Validation}

4.1. Data Collection. To calibrate and validate the proposed model, the data of the three straddling work zones were collected. The data collection time was from 17:30 to 19:30 on weekdays in Chengdu, Sichuan Province, China. Two UAVs (Unmanned Aerial Vehicles) were used to record the traffic information of the three intersections. Meanwhile, the signal information and the geometry information of the work zone and intersections were surveyed. All the three intersections had four legs, while the different intersections may have different numbers of approach and exit lanes. The work zones also have different sizes. For the basic geometry information data of the three intersections and work zones, see Table 1 . The traffic flow data are displayed in Table 2. In Table $2, t_{f}$ denotes the average passing time of the leading vehicle, $t_{o}$ denotes the average passing time of intermediate vehicles, $T$ denotes the signal phase period, $G$ denotes the green light duration of the signal phase, $R$ denotes the red light duration of the signal phase, and $Y$ denotes the amber light duration of the signal phase.

4.2. Calibration and Validation Results. The method based on the macroscopic traffic data is applied to calibrate the proposed model. This method was widely adopted to calibrate the traffic flow models $[5,10,15,25-27]$. The calibration method is to solve a nonlinear programming problem in which Thiel's $U$ function is taken as the objective function, the model parameters are the independent variables, and the feasible ranges of the model parameters are the constraints [16, 28-31] (see Table 3). The objective function in the used calibration method is as follows:

$$
U=\frac{\sqrt{(1 / N) \sum_{k=1}^{N}\left(\widehat{z}_{k}-z_{k}\right)^{2}}}{\sqrt{(1 / N) \sum_{k=1}^{N}\left(\widehat{z}_{k}\right)^{2}}+\sqrt{(1 / N) \sum_{k=1}^{N}\left(z_{k}\right)^{2}}},
$$

where $z_{k}$ denotes the real value of the evaluation variable for the $k^{\text {th }}$ observation, $\widehat{z}_{k}$ denotes the estimated value of $z_{k}$, and $N$ denotes the total number of observations.

In equation (11), the evaluation variable $z_{k}$ has six alternatives: the number of total vehicles $N_{t}$, the number of cars $N_{c}$, the average travel times of the leading vehicles and all vehicles $T_{f}$ and $T_{a}$, and the average speeds of the leading vehicles and all vehicles $S_{f}$ and $S_{a}$. In all five collected observations, four of them will be used to calibrate the proposed model, and the remaining one will be used to validate the model. Genetic Algorithm (GA) embedded in the GA toolbox of MATLAB is adopted to solve the nonlinear programming problem [32]. To obtain the best results, the GA function is run for ten times. In the end, the optimal parameter values for the proposed model are obtained (see Table 3).

The three MOE (measure of effectiveness) indexes are used to evaluate the performance of the proposed model: mean error (me), mean absolute error (mae), and mean absolute relative error (MARE). They are calculated as follows $[26,33]$ : 
TABLe 1: Collected intersection and work zone data.

\begin{tabular}{lccc}
\hline & Site 1 & Site 2 & Site 3 \\
\hline Survey time & $17: 30-19: 30$ & $17: 30-19: 30$ & 4 \\
Number of the approach lanes of the entrance 1 & 2 & Blocked & 4 \\
Number of the exit lanes of the entrance 1 & 2 & 3 & Blocked \\
Number of the approach lanes of the entrance 3 & 2 & 3 & 3.25 \\
Number of the exit lanes of the entrance 3 & 2 & Blocked & 3.25 \\
Width of the approach lane $(\mathrm{m})$ & 3.25 & Blocked \\
Width of the exit lane $(\mathrm{m})$ & &
\end{tabular}

TABLE 2: Used traffic data of straddling work zone in signalized intersection.

\begin{tabular}{lcccccccccc}
\hline Site no. & Approach no. & Cars (vehs) & Buses (vehs) & Articulated buses (vehs) & $t_{f}(\mathrm{~s})$ & $t_{o}(\mathrm{~s})$ & $T(s)$ & $G(\mathrm{~s})$ & $R(\mathrm{~s})$ & $Y(s)$ \\
\hline 1 & 1 & 23 & 2 & 2 & 16.4 & 21.5 & 115 & 50 & 62 & 3 \\
2 & 3 & 34 & 4 & 3 & 18.3 & 24.4 & 115 & 50 & 62 & 3 \\
3 & 1 & 36 & 2 & 1 & 15.7 & 19.9 & 99 & 46 & 50 & 3 \\
3 & 1 & 62 & 7 & 0 & 10.7 & 11.3 & 133 & 95 & 45 & 3 \\
\hline
\end{tabular}

TABLE 3: Feasible ranges of the parameters and the optimal values.

\begin{tabular}{lcccccccccc}
\hline Model parameters & $a_{\max }$ & $b$ & $\tau_{i}$ & $A^{r}$ & $A^{p}$ & $A^{b}$ & $A^{\text {fr }}$ & $\Delta d$ & $B_{j}$ & $B_{i}$ \\
\hline Feasible range & $0 \sim 5$ & $0 \sim 5$ & $0 \sim 5$ & $0 \sim 15$ & $0 \sim 15$ & $0 \sim 15$ & $0 \sim 15$ & $0 \sim 1$ & $0 \sim 5$ & $0 \sim 5$ \\
Optimal values for cars & 2.45 & 3 & 0.994 & 4.346 & 7.040 & 7.194 & 2.917 & 0.43 & 1.882 & 0.690 \\
Optimal values for buses & 2.33 & 2.76 & 1.491 & 6.308 & 3.647 & 7.377 & 3.609 & 0.64 & 1.574 & 1.547 \\
\hline
\end{tabular}

$$
\begin{aligned}
\mathrm{ME} & =\frac{1}{N} \sum_{k=1}^{N}\left(z_{r, k}-z_{s, k}\right), \\
\mathrm{MAE} & =\frac{1}{N} \sum_{k=1}^{N}\left|z_{r, k}-z_{s, k}\right|, \\
\mathrm{MARE} & =\frac{1}{N} \sum_{k=1}^{N}\left(\frac{\left|z_{r, k}-z_{s, k}\right|}{z_{r, k}}\right) .
\end{aligned}
$$

The calibration and validation results are displayed in Table 4. From Table 4, it can be observed that the MARE values for the six variables are not more than 0.05 . In particular, the MAE of $N_{c}$ is around 2, revealing that the difference between the simulated vehicle number and real vehicle number is also very small. All of the results indicate that the proposed model has high performance in replicating the traffic flow of the intersection with the straddling work zone.

\section{Simulations and Results Analysis}

5.1. Simulation Setup. In the simulations, an intersection with a straddling work zone is set up, as shown in Figure 4. The intersection has the same scenery and signal design with Site 1 in the collected data. Both cars and buses are contained in the simulations. The original generation probability of the vehicle type is subject to the type proportions of the field data of Site 1. The simulation time step is set as 1 second. The outputs of the simulation have both the microscopic and macroscopic traffic data. The microscopic data includes the speed, position, and acceleration of vehicles at each time step. The macroscopic data are from the aggregated results of the microscopic outputs including the average travel time, average speed, and saturation flow rate.

5.2. Distance from Lower Edge of the Work Zone to the Median Divider of the Road $(W)$. Figure 5 displays the change of the speed and the number of the passed vehicles in a signal with the variation of $W$ in Figure 4 . It can be observed from Figure 5 that when $0 \mathrm{~m} \leq W<7 \mathrm{~m}$, the larger the distance of the upper edge of the work zone to the median divider of the road is, the larger the passing area is. The relationship of $N_{a}^{f i t}$ and $W$ is linear. When $W$ is close to or greater than the width of the left entrance, that is, $W \geq 7 \mathrm{~m}$, the capacity of the intersection is close to the normal intersection. At this time, the work zone is located on the road segment and the influence of the work zone on the intersection can be neglected, so $N_{a}^{f i t}$ remains unchanged. In addition, the result shows that the average passing speed of the vehicles increases with the increment of $W$. The reason is that when the value of $W$ increases, more road resources can be provided for the vehicles to pass. The interactions between the vehicles and their coming vehicles are reduced, so the average passing speed will increase.

5.3. Width of the Unoccupied Lane (D). In the straddling work zone in Figure 6(a), D denotes the width of the open area in the work zone. The volume of traffic and the average passing speed of the vehicles in a certain green time are simulated by changing the value of $D$. As shown in 
TABLE 4: Evaluation results of work zone social force model.

\begin{tabular}{|c|c|c|c|c|c|c|}
\hline & \multicolumn{3}{|c|}{ Calibration results } & \multicolumn{3}{|c|}{ Validation results } \\
\hline & $\mathrm{ME}$ & MAE & MARE & ME & MAE & MARE \\
\hline$N_{t}$ & -0.25 & 1.15 & 0.02 & -0.20 & 0.62 & 0.02 \\
\hline$N_{c}$ & 0.16 & 1.72 & 0.04 & 0.15 & 1.50 & 0.03 \\
\hline$T_{f}$ & 0.51 & 0.54 & 0.02 & 0.32 & 0.26 & 0.01 \\
\hline$S_{f}$ & -0.09 & 0.11 & 0.05 & -0.12 & 0.13 & 0.04 \\
\hline$T_{a}$ & -0.08 & 1.15 & 0.05 & -0.10 & 1.20 & 0.05 \\
\hline$S_{a}$ & -0.09 & 0.06 & 0.03 & -0.11 & 0.05 & 0.04 \\
\hline
\end{tabular}

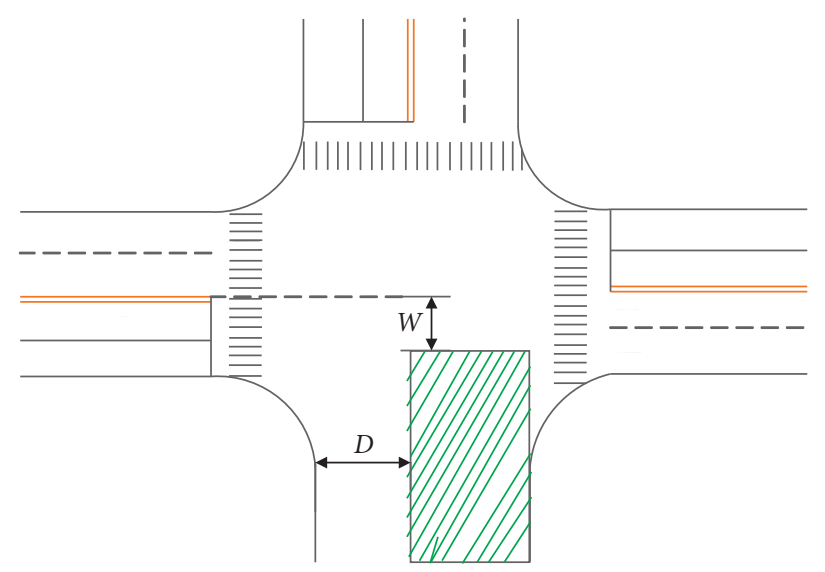

FIGURE 4: The intersection simulation setup.

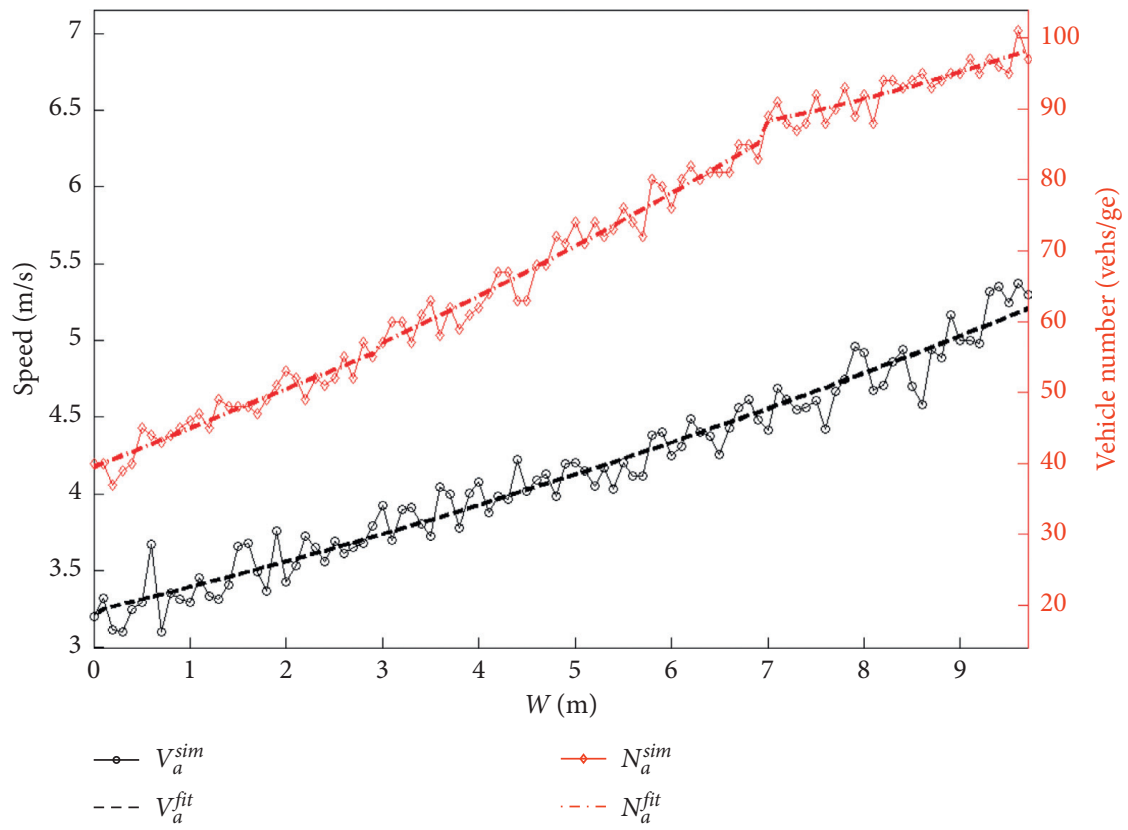

FIgURE 5: Diagrams under different $W$ of the straddling work zone intersection.

Figure 6(b), from the initial state, the length of the work zone $D=0$, that is, the lower part, is fully closed. The numbers of approach lanes and exit lanes in the work zone are 2, and the number of non-motor-vehicle lanes is 1 , so there are 3 lanes in one direction. When $0 \leq D<6.5 \mathrm{~m}$, the half of the road is occupied by the work zone. In this situation, the traffic volume and average speed increase fast with the increment of $D$. When $D \geq 6.5 \mathrm{~m}$, although the traffic volume and average speed also increase when $D$ becomes bigger, the increasing slope is smaller than the case of $0 \leq D<6.5 \mathrm{~m}$. 


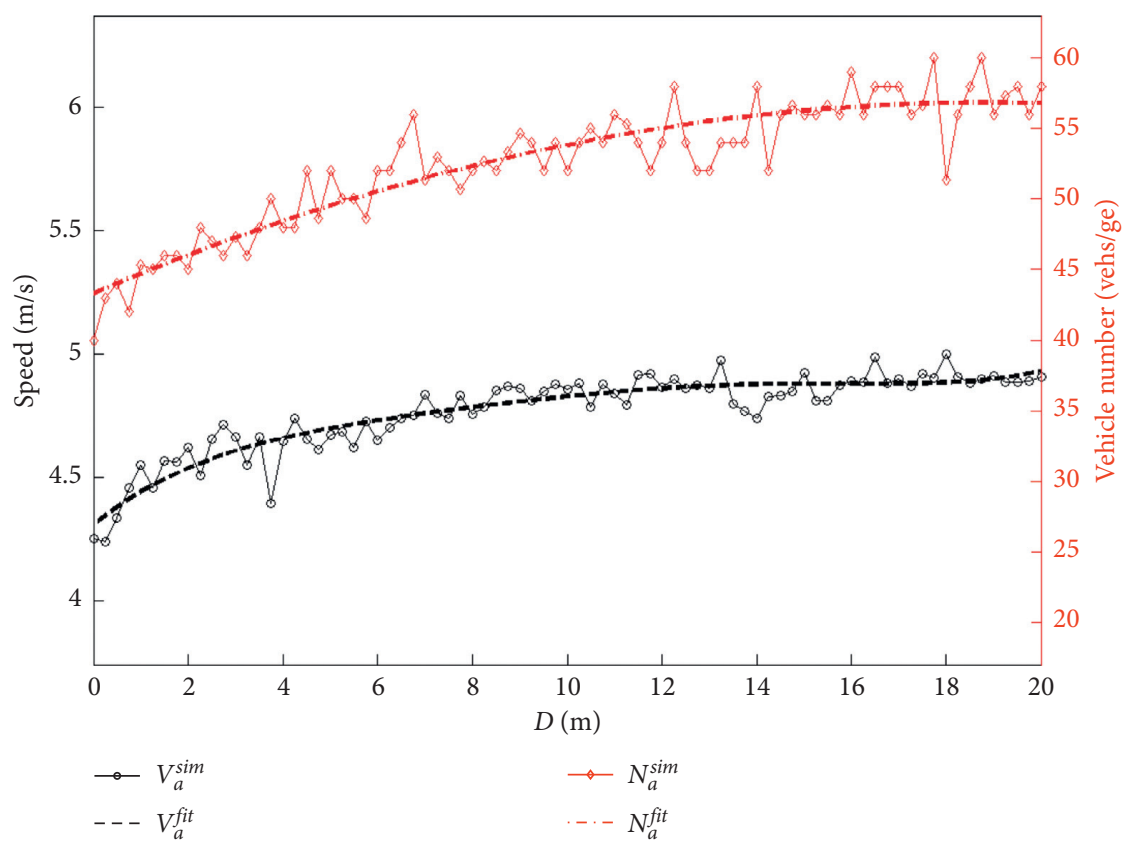

Figure 6: Diagrams under different $D$ of the straddling work zone intersection.

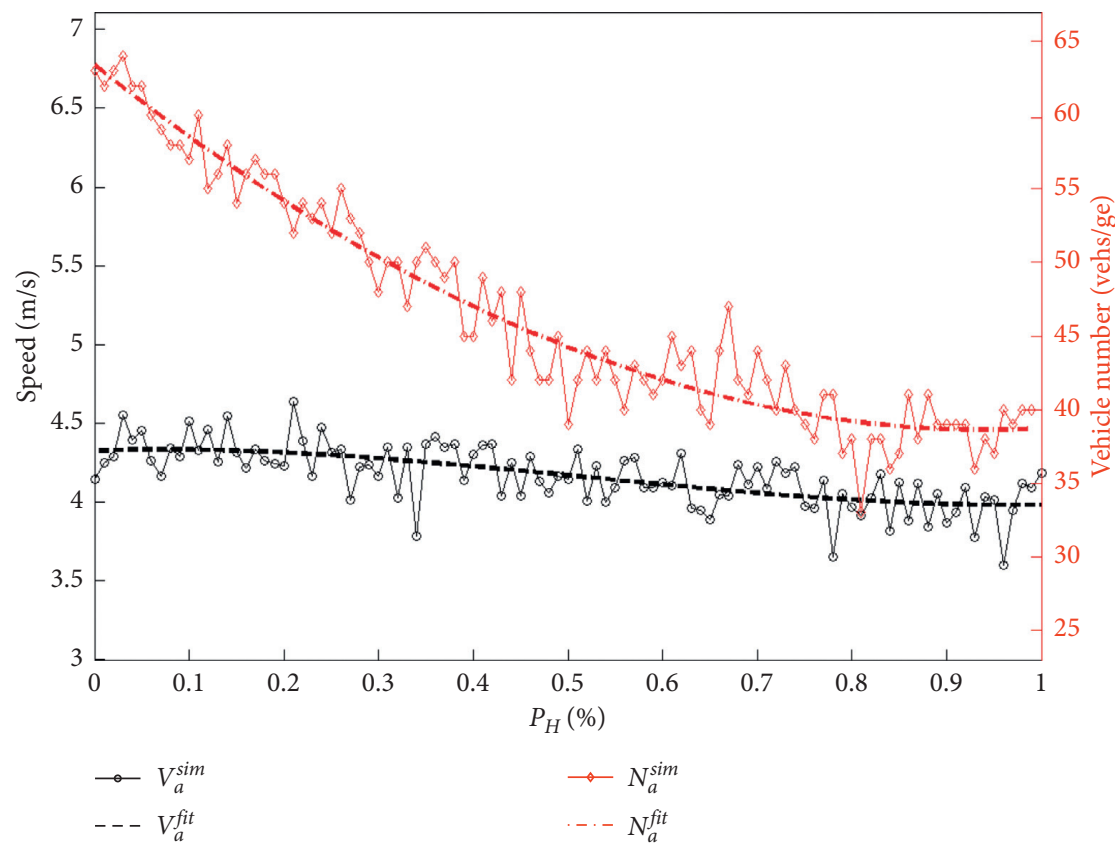

Figure 7: Diagrams under different $P_{H}$ of work zone.

5.4. Proportion of Large Vehicles $\left(P_{H}\right)$. Figure 7 illustrates the trend of traffic flow and average speed in the case of gradually increasing the proportion of large vehicles. In our simulation, the value of $P_{H}$ varies between 0 and 1 . As shown in Figure 7, it can be observed that when $0 \leq P_{H}<0.55$, the number of vehicles passing through the intersection and their average speed both decrease significantly with the increment of large vehicles. When $P_{H} \geq 0.55$, if the proportion of large vehicles continues to increase, the decrement of the traffic volume is no longer significant and tends to be stable. The traffic volume at this time reaches the minimum value. The large vehicles have slower speed and will occupy more space, which leads to a decrement of the average speed of the traffic.

5.5. Proportion of Left-Turn Vehicles $\left(P_{L}\right)$. Figure 8 displays the impact of the proportion of the left-turn vehicles on the traffic flow of the intersection with a straddling work 


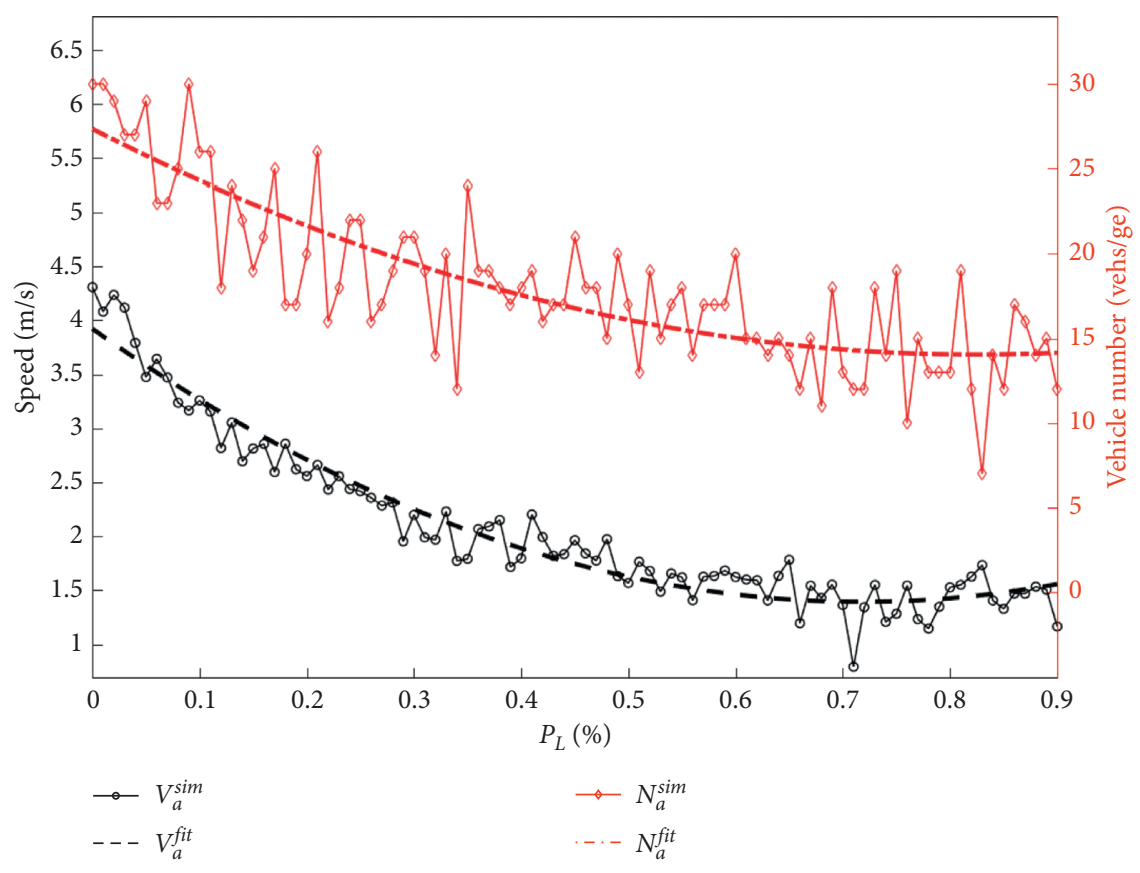

Figure 8: Diagrams under different $P_{L}$ of work zone.

zone. In the simulations, the proportion of the left-turn vehicles increases from 0 to 0.9 gradually. It can be observed that the speed and the number of passed vehicles will decline with the increment of the proportion of the left-turn vehicles. The reason is that the increment of left-turn vehicles will intensify the interactions of the through-movement and left-turn movement. Besides, when $P_{L}$ is less than 0.6 , the vehicle number and speed decrease significantly, while when $P_{L}$ is greater than 0.6 , the decreasing trend is mitigated. The reason for this phenomenon is that the left-turn vehicles have become the main component of the traffic flow when $P_{L}>0.6$, and increasing $P_{L}$ will reduce the mixture degree and interactions of the left-turn and through vehicles.

5.6. Analysis on the Three-Leg Intersection. In order to verify the applicability of the proposed model in other scenarios, we carried out simulations for a three-leg intersection with a straddling work zone, as displayed in Figure 9. The parameters, such as the number of lanes and lane width, are taken to be the same as those in Section 5.1 .

Figure 10 illustrates the simulation results for the three-leg intersection with a straddling work zone. Figures 10(a)-10(d), respectively, exhibit the impacts of the distance from the lower edge of the work zone to the median divider of the road $(W)$, width of the unoccupied lane $(D)$, proportion of large vehicles $\left(P_{H}\right)$, and proportion of left-turn vehicles $\left(P_{L}\right)$ on the traffic flow. It can be observed that the proposed model is also applicable to the three-leg intersection with a straddling work zone. The impact patterns of the above variables on traffic flow are much similar to that of the crossing intersection, while the tendency-changing thresholds are different for the two

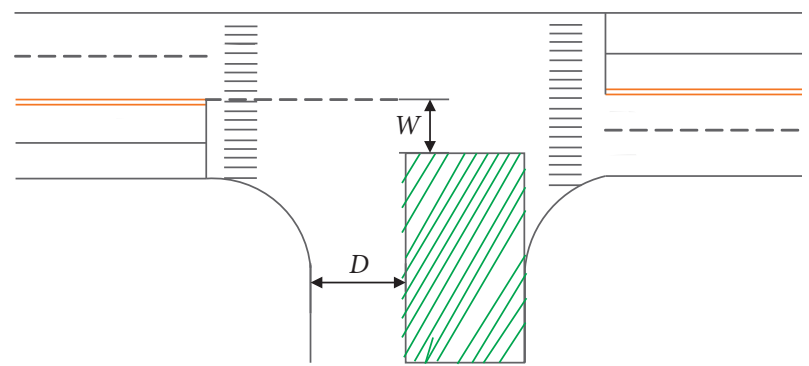

FIGURE 9: The three-leg intersection with a straddling work zone.

types of intersection, and the impacts of the large vehicles and left-turning vehicles on traffic flow are not as significant as that of the crossing intersection.

\section{Conclusions and Future Work}

A large number of work zones exist on urban arterials in many countries, which have a great impact on the urban traffic. However, studies on the urban arterial work zones are very limited, especially on the work zones at the intersections. Based on the analysis of the traffic flow characteristics of the work zone, we propose a social forcebased model for a specific type of work zone which straddles on an intersection and a segment, namely, the straddling work zone. In order to calibrate and validate the proposed model, the data of the three straddling work zones were collected in Chengdu, China. The following are the main conclusions drawn in this paper:

(1) The proposed model can describe the traffic flow characteristics of the intersection with a straddling 


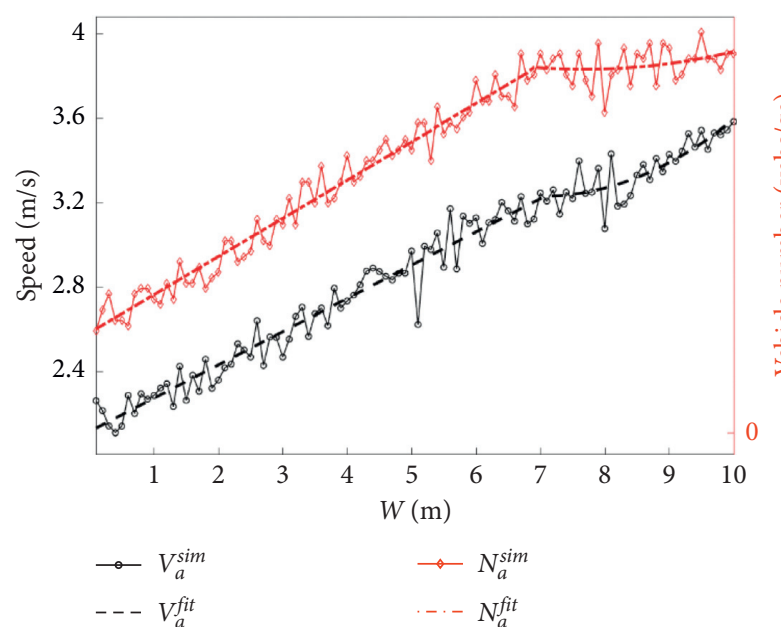

(a)

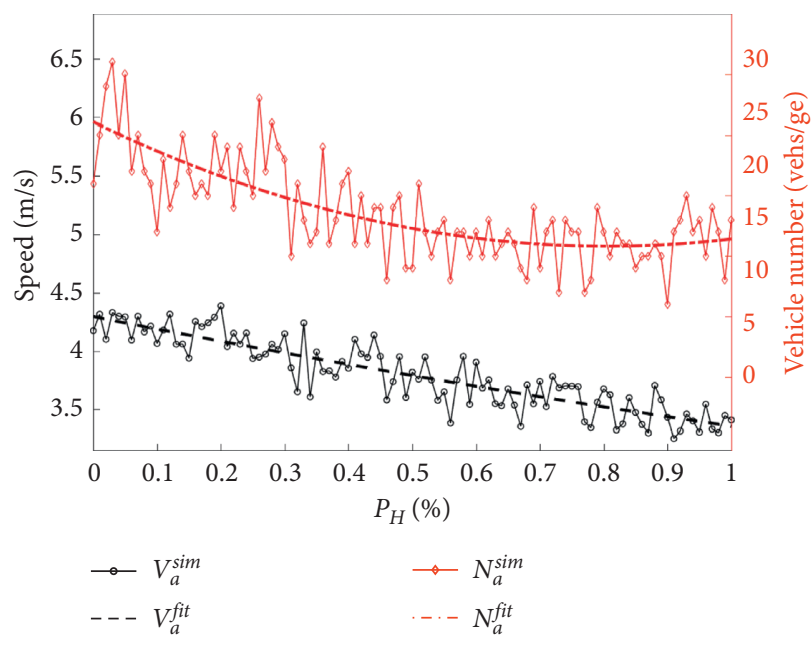

(c)

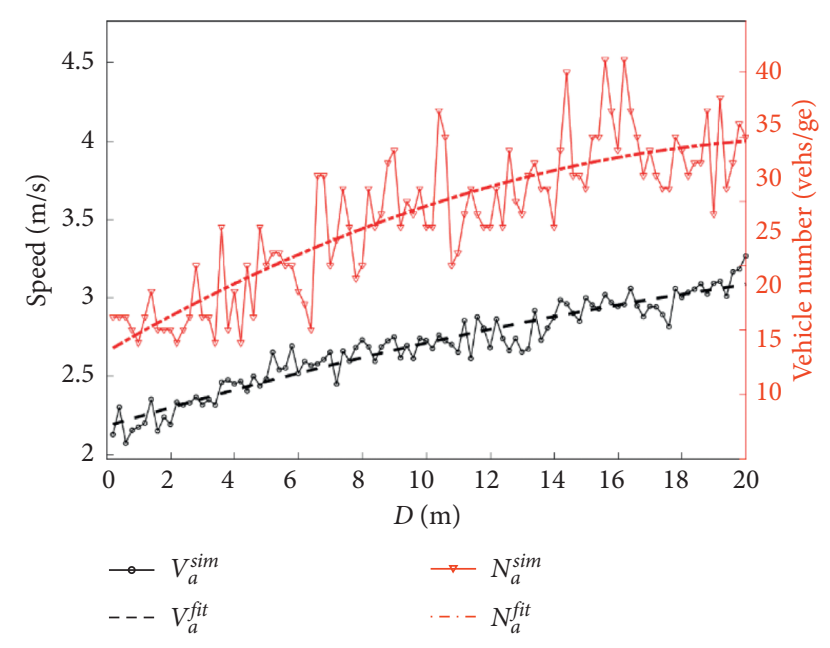

(b)

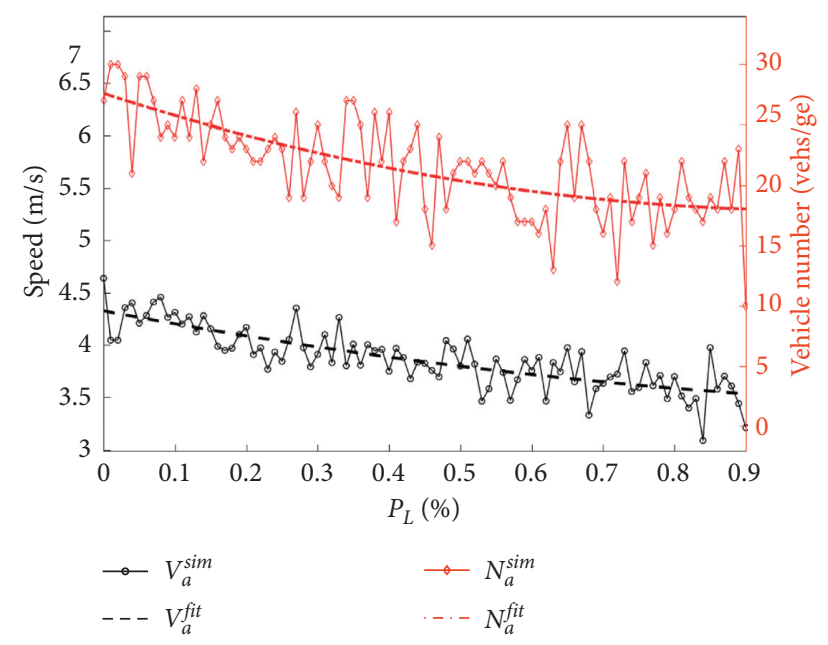

(d)

Figure 10: Simulation results for the three-leg intersection. (a) $W$. (b) $D$. (c) $P_{H}$. (d) $P_{L}$.

work zone with high performance. The MARE values of the traffic volume, the average speed, and the time passing through the work zone are less than $10 \%$.

(2) When the work zone exists, the traffic volume is inversely proportional to the distance from the lower edge of the work zone to the median divider of the road. The simulations show that the volume becomes larger as $W$ increases and remains basically unchanged when $W$ is greater than half of the intersection.

(3) For the ratio of large vehicles and the ratio of leftturn vehicles, there is a value $P$ which makes the traffic volume become obviously decreased with the increment of $P_{H}$ when $0 \leq P_{H}<P$, and the traffic volume tends to be stable when $P_{H} \geq P$.

(4) The proposed model is also applicable to the threeleg intersection with a straddling work zone, and the influences of several key variables are similar to that of the crossing intersections.
However, this study also has some limitations. First, based on the proposed model, the signal optimization problem for the intersection with a straddling work zone can be conducted in the future, which will help to reduce the negative impact of the work zone on intersection traffic. Second, more work zone types will be explored and their influences on traffic will be discussed in the future.

\section{Data Availability}

The data used to support the findings of this study are included within the article.

\section{Conflicts of Interest}

The authors declare that they have no conflicts of interest to declare. 


\section{Acknowledgments}

This work was supported by Soft Science Project of Chengdu City (Grant no. 2019RK0000054ZF) and Key Research and Development Project of Sichuan Province (Grant no. 19ZDYF2068).

\section{References}

[1] K. Heaslip, A. Kondyli, D. Arguea, L. Elefteriadou, and F. Sullivan, "Estimation of freeway work zone capacity through simulation and field data," Transportation Research Record: Journal of the Transportation Research Board, vol. 2130, no. 1, pp. 16-24, 2009.

[2] J. Weng and X. Yan, "New methodology to determine work zone capacity distribution," Transportation Research Record: Journal of the Transportation Research Board, vol. 2461, no. 1, pp. 25-31, 2014.

[3] M. Chitturi and R. Benekohal, "Work zone queue length and delay methodology," Transportation Letters, vol. 2, no. 4, pp. 273-283, 2010.

[4] J. Weng, J.-Z. Zhu, X. Yan, and Z. Liu, "Investigation of work zone crash casualty patterns using association rules," Accident Analysis \& Prevention, vol. 92, pp. 43-52, 2016.

[5] J. Weng, G. Li, and Y. Yu, "Time-dependent drivers' merging behavior model in work zone merging areas," Transportation Research Part C: Emerging Technologies, vol. 80, pp. 409-422, 2017.

[6] N. N. Sze and Z. Song, "Factors contributing to injury severity in work zone related crashes in New Zealand," International Journal of Sustainable Transportation, vol. 13, no. 2, pp. 148-154, 2019.

[7] D. Yang, X. Zhou, G. Su, and S. Liu, "Model and simulation of the heterogeneous traffic flow of the urban signalized intersection with an island work zone," IEEE Transactions on Intelligent Transportation Systems, vol. 20, no. 5, pp. 17191727, 2019.

[8] E. Swansen, M. Knodler, and J. Kennedy, "Determining work zone crash causation using linked crash and citation data," in Proceedings of the Managing Operational Performance Exceeding Expectations. 2012 ITE Technical Conference and Exhibit, Pasadena, CA, USA, March 2012.

[9] A. Karim and H. Adeli, "Radial basis function neural network for work zone capacity and queue estimation," Journal of Transportation Engineering, vol. 129, no. 5, pp. 494-503, 2003.

[10] Q. Meng and J. Weng, "Cellular automata model for work zone traffic," Transportation Research Record: Journal of the Transportation Research Board, vol. 2188, no. 1, pp. 131-139, 2010.

[11] Y. Li and Y. Bai, "Fatal and injury crash characteristics in highway work zones," in Proceedings of the Transportation Research Board 87th Annual Meeting, Washington, CO, USA, Janaury 2006.

[12] K. Shaaban and D. Elnashar, "Evaluation of work zone strategies at signalized intersections," Journal of Traffic and Logistics Engineering, vol. 1, no. 2, pp. 202-206, 2013.

[13] S. Washburn, T. Hiles, and K. Heaslip, "Impact of lane closures on roadway capacity: arterial work zone capacity (Part B)," University of Florida, Gainesville, FL, USA, 2008.

[14] D. Yang, L. Zhu, L. Ma, and R. Sun, "Model for the capacity of the urban signal intersection with work zone," Journal of Advanced Transportation, vol. 50, no. 7, pp. 1506-1519, 2016.
[15] Y. Hou, P. Edara, and C. Sun, "Traffic flow forecasting for urban work zones," IEEE Transactions on Intelligent Transportation Systems, vol. 16, no. 4, pp. 1761-1770, 2015.

[16] D. N. Huynh, M. Boltze, and A. T. Vu, "Modelling mixed traffic flow at signalized IntersectionUsing social force model," Journal of the Eastern Asia Society for Transportation Studies, vol. 10, pp. 1734-1749, 2013.

[17] L. X. Nguyen, S. Hanaoka, and T. Kawasaki, "Describing nonlane-based motorcycle movements in motorcycle-only traffic flow," Transportation Research Record: Journal of the Transportation Research Board, vol. 2281, no. 1, pp. 76-82, 2012.

[18] A. Sadegh, A. E. Radwan, and N. Rouphail, "ARTWORK: a simulation model of urban arterial work zones," Transportation Research Record: Journal of the Transportation Research Board, vol. 1163, pp. 1-3, 1988.

[19] C. Joseph, E. Radwan, and N. M. Rouphail, "Work zone analysis model for the signalized arterial," Transportation Research Record: Journal of the Transportation Research Board, vol. 1194, 1988.

[20] A. Tsyganov, R. Machemehl, and K. Liapi, "Identification of traffic control problems on urban arterial work zones," Technical Report FHWA/TX-04/4266-1, University of Texas, Austin, TX, USA, 2003.

[21] A. R. Tsyganov, D. N. Mohan, and R. B. Machemehl, "Analysis of accident statistics on urban arterial street work zones," in Proceedings of the 12th World Congress on Intelligent Transport Systems, San Francisco, CA, USA, November 2005.

[22] K. Heaslip, M. Jain, and L. Elefteriadou, "Estimation of arterial work zone capacity using simulation," Transportation Letters, vol. 3, no. 2, pp. 123-134, 2011.

[23] NYSDT, "Highway design manual," Maintenance and Protection of Traffic in Highway Work Zones, New York State Department of Transportation, Albany, NY, USA, 2006.

[24] P. G. Gipps, "A model for the structure of lane-changing decisions," Transportation Research Part B: Methodological, vol. 20, no. 5, pp. 403-414, 1986.

[25] E. Brockfeld, R. D. Kühne, and P. Wagner, "Calibration and validation of microscopic models of traffic flow," Transportation Research Record: Journal of the Transportation Research Board, vol. 1934, no. 1, pp. 179-187, 2005.

[26] Q. Meng and J. Weng, "An improved cellular automata model for heterogeneous work zone traffic," Transportation Research Part C: Emerging Technologies, vol. 19, no. 6, pp. 1263-1275, 2011.

[27] H. Rakha and M. Arafeh, "Calibrating steady-state traffic stream and car-following models using loop detector data," Transportation Science, vol. 44, no. 2, pp. 151-168, 2010.

[28] B. Anvari, M. G. H. Bell, A. Sivakumar, and W. Y. Ochieng, "Modelling shared space users via rule-based social force model," Transportation Research Part C: Emerging Technologies, vol. 51, pp. 83-103, 2015.

[29] L. Fei, H. B. Zhu, and X. L. Han, "Analysis of traffic congestion induced by the work zone," Physica A: Statistical Mechanics and Its Applications, vol. 450, pp. 497-505, 2016.

[30] D. Helbing and B. Tilch, "Generalized force model of traffic dynamics," Physical Review E, vol. 58, no. 1, pp. 133-138, 1998.

[31] F. E. Gunawan, "Two-vehicle dynamics of the car-following models on realistic driving condition," Journal of Transportation Systems Engineering and Information Technology, vol. 12, no. 2, pp. 67-75, 2012.

[32] P. J. Jin, D. Yang, and B. Ran, "Reducing the error accumulation in car-following models calibrated with vehicle trajectory data," IEEE Transactions on Intelligent Transportation Systems, vol. 15, no. 1, pp. 148-157, 2014. 
[33] V. Punzo and F. Simonelli, "Analysis and comparison of microscopic traffic flow models with real traffic microscopic data," Transportation Research Record: Journal of the Transportation Research Board, vol. 1934, no. 1, pp. 53-63, 2005. 